\title{
Single-Size Thermometric Measurements on a Size Distribution of Neutral Fullerenes
}

\author{
C. Cauchy, ${ }^{1}$ J. M. Bakker, ${ }^{2}$ Y. Huismans, ${ }^{3}$ A. Rouzée, ${ }^{3,4}$ B. Redlich, ${ }^{2}$ A. F. G. van der Meer, ${ }^{2,5}$ C. Bordas, ${ }^{1}$ \\ M. J. J. Vrakking, ${ }^{3,4}$ and F. Lépine ${ }^{1, *}$ \\ ${ }^{1}$ Institut Lumière Matière, Université Lyon 1, CNRS, UMR 5306, 10 rue Ada Byron, 69622 Villeurbanne Cedex, France \\ ${ }^{2}$ FOM Institute for Plasma Physics Rijnhuizen, Edisonbaan 14, 3439 MN Nieuwegein, Netherlands \\ ${ }^{3}$ FOM-Institute AMOLF, Science Park 104, 1098 XG Amsterdam, Netherlands \\ ${ }^{4}$ Max-Born-Institut, Max Born Straße 2A, D-12489 Berlin, Germany \\ ${ }^{5}$ Institute for Molecules and Materials, FELIX Facility, Radboud University Nijmegen, \\ Toernooiveld 7, 6525 ED Nijmegen, Netherlands
}

(Received 20 June 2012; published 6 May 2013)

\begin{abstract}
We present measurements of the velocity distribution of electrons emitted from mass-selected neutral fullerenes, performed at the intracavity free electron laser FELICE. We make use of mass-specific vibrational resonances in the infrared domain to selectively heat up one out of a distribution of several fullerene species. Efficient energy redistribution leads to decay via thermionic emission. Time-resolved electron kinetic energy distributions measured give information on the decay rate of the selected fullerene. This method is generally applicable to all neutral species that exhibit thermionic emission and provides a unique tool to study the stability of mass-selected neutral clusters and molecules that are only available as part of a size distribution.
\end{abstract}

DOI: 10.1103/PhysRevLett.110.193401

PACS numbers: $36.40 .-\mathrm{c}, 33.80 .-\mathrm{b}$

The stability of an excited polyatomic system is determined by the existence of a wide variety of decay pathways that may occur simultaneously. For a sufficiently complex system, the decay can generally be described as a statistical process because of the efficient couplings between the degrees of freedom. Such mechanisms are of wide interest, as they appear, for instance, in biologically relevant species. Therefore, the determination of the decay rate, measuring the stability of the molecule, is crucial for both fundamental and application purposes. However, this decay rate is often difficult to access experimentally, first because the decay of a molecule may occur through channels that are not detected. Moreover, this quantity depends strongly on the exact species and therefore, it relies on our ability to produce a pure sample. For instance, in most cluster spectroscopies, preselection of a given mass is performed for ionic species. However, for small clusters or molecules neutral and charged species have very different properties and specific experiments have to be performed on both.

Alternatively, many experiments are using a molecular beam containing a unique mass of neutral species. This considerably constrains the variety of systems that can be studied and has been one of the many motivations for studying fullerenes such as $\mathrm{C}_{60}$. The methodologies developed for fullerenes are often taken as initial demonstrations for future experiments in cluster and molecular physics [1]. Fullerenes are also relevant objects to address questions on photoinduced energy flow, stability, and decay mechanisms in polyatomic systems [2]. In the past decades it has been shown that highly excited fullerenes undergo delayed emission of electrons, dissociation via sequential loss of $\mathrm{C}_{2}$ fragments and radiative cooling [3]. Note that, although electronic and vibrational spectroscopy has been performed for many fullerenes, accurate knowledge on their decay mechanisms has essentially been limited to $\mathrm{C}_{60}$ and $\mathrm{C}_{70}$ owing to mass selection restrictions.

In this Letter we propose a novel method to perform thermometric measurements on mass-selected highly excited neutral species. This method is based on the combination of time-resolved velocity map imaging spectrometry with the new intracavity free electron laser FELICE [4]. As an illustration we present results obtained on fullerenes, although it must be emphasized that the method can be used for many other cluster or molecular species. FELICE delivers intense tunable far-IR light and offers the possibility to selectively excite resonances specific to a given fullerene mass. In addition, the intracavity arrangement allows exposing free molecules or clusters to the intense IR radiation without strong focusing, ensuring a long interaction time between the molecular beam and the FEL light. With efficient internal vibrational energy redistribution (IVR) and the long interaction time, a high internal energy of the resonantly excited molecules is reached. Because of the resonant character of the excitation the selected species is highly excited while other species remain essentially cold. The excited molecules consequently undergo thermionic emission. The 3D velocity distribution of the emitted electrons is measured as a function of time using a velocity map imaging spectrometer. These measurements lead to direct information on the temperature and the decay rate of the selected molecule.

Our experimental setup is sketched in Fig. 1. A velocity map imaging (VMI) spectrometer is integrated into the cavity of the FELICE FEL. A ceramic oven containing a 


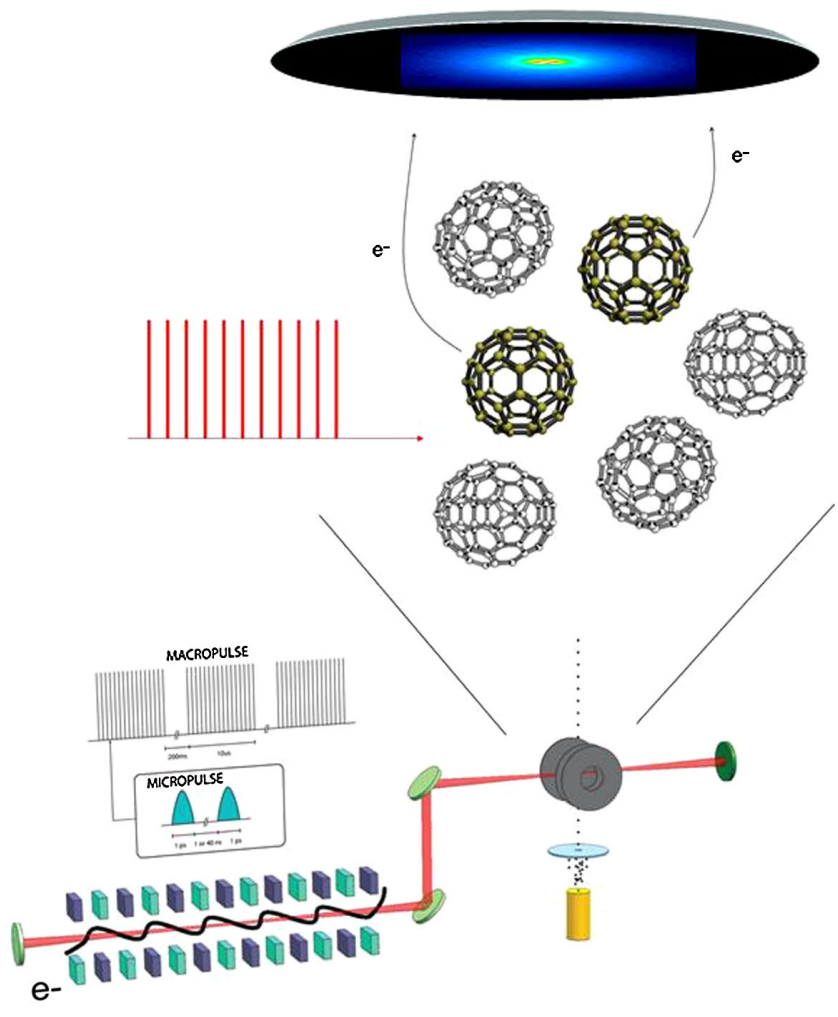

FIG. 1 (color online). Schematic of our experimental setup. A molecular beam composed of a mixture of fullerenes interacts with the FELICE light tuned to a vibrational resonance specific to a given fullerene mass. The selected fullerene is heated up and ionized by IR-REMPI. The emitted electrons are recorded with a time-gated velocity map imaging spectrometer. The electron kinetic energy spectrum gives access to the temperature of the selected cluster.

mixture of $\mathrm{C}_{60}, \mathrm{C}_{70}$ and heavier fullerenes $\left(\mathrm{C}_{60}[50 \%], \mathrm{C}_{70}\right.$ [30\%], $\mathrm{C}_{84}$ [8\%], other masses $>\mathrm{C}_{70}[12 \%]$ ) is brought to $500 \mathrm{~K}$ in order to produce a molecular beam crossing the FELICE IR beam at right angles in the extraction region of the VMI spectrometer. The spectrometer axis is perpendicular both to the FELICE optical beam and molecular beam axes. The velocity of the molecules in the jet is typically $160 \mathrm{~m} / \mathrm{s}$, allowing their observation over several tens of $\mu \mathrm{s}$ before leaving the interaction region. In FELICE, tunable far-IR radiation is generated by electron bunches passing through an undulator structure, and is enclosed in a four-mirror cavity, containing a waist characterized by a Rayleigh range $z_{0}=55 \mathrm{~mm}$ in a section designed for the installation of intracavity experiments. The temporal structure of the light bursts consists of micropulses with a repetition rate of either $1 \mathrm{GHz}$ or $16.7 \mathrm{MHz}$, which are grouped in macropulses with a typical duration of $5 \mu$ s operating at a repetition rate of 5 or $10 \mathrm{~Hz}$. FELICE operates between 100 and $3500 \mathrm{~cm}^{-1}(3-100 \mu \mathrm{m})$, with micro-pulse energies up to $1.5 \mathrm{~mJ}$. The typical peak intensity used in the experiments was $10^{11} \mathrm{~W} / \mathrm{cm}^{2}$. The VMI spectrometer follows the standard design introduced by
Parker et al. [5]. In order to follow the time evolution of the kinetic energy electron spectra, pulsed detection is used by means of a high-voltage (HV) switch applied to the detector and synchronized to the FELICE macro-pulses. The PSD consists of a pair of microchannel plates followed by a phosphor screen and a CCD camera. The photoexcitation by FELICE over the $5 \mu$ s duration of the FELICE macropulses produces an overlap of the time-of-flight peaks of the various ions. In order to record the ion mass spectrum and the ion yield of the different masses, a pulsed extraction voltage is applied to the VMI electrodes.

Time-resolved electron velocity distributions were measured at various time delays. The analysis of such measurements and the information contained in the timedependent spectra were explained in a previous article [6]. The key idea is that the decay rate of the molecule varies very rapidly with internal energy, so that electrons detected in a well-defined time window are necessarily emitted from a cluster having a well-defined internal energy. Clusters having lower internal energy will decay at a much later time delay while systems having higher internal energy have already decayed when the measurement has occurred. In the statistical theory of finite size systems [7], the internal energy $E$ of the cluster determines its microcanonical temperature $T_{\mu}(E)$ defined by $1 / k_{B} T_{\mu}(E)=$ $\left.(\partial \ln [\rho(x)] / \partial x)\right|_{x=E}$, where $\rho(x)$ corresponds to the density of states at the internal energy $x$. For a neutral system, the electron kinetic energy spectrum measured at a given time delay $t_{m}$ can be written as

$$
P\left(\varepsilon, t_{m}\right) \propto \exp \left(\frac{-\varepsilon}{k_{B} T}\right),
$$

where $\varepsilon$ is the electron kinetic energy, $T$ is the so-called daughter temperature of the cluster defined by $T=$ $T_{\mu}(E-I P)$ where $I P$ corresponds to the ionization potential. Therefore, the time-dependent electron spectrum allows a direct measurement of temperature $T$. This thermometric measurement gives access to the relation between the temperature and emission time delay $t_{m}$. As a consequence, it measures the decay rate $K$ following the relation $K(T) \approx 1 / t_{m}$. This rate corresponds to the total decay rate of the molecule. In the case of fullerenes, it is the sum of three dominant contributions, namely, thermionic emission, fragmentation by loss of $\mathrm{C}_{2}$ fragments, and radiative cooling.

We have previously applied this method in the case of $\mathrm{C}_{60}$ excited with a ns laser pulse in the UV-vis wavelength range [8]. The same approach has now been applied to the measurements obtained with FELICE. The FEL was tuned to resonantly excite the degenerated $F_{1 u}$ vibrational mode at $525 \mathrm{~cm}^{-1}$ leading to ionization of $\mathrm{C}_{60}$ [9]. At this frequency no other fullerene is excited. After the resonant absorption of a single photon, the vibrational couplings allow efficient energy redistribution over the whole molecular structure before the subsequent absorption of a photon from the next micropulse. During the macropulse, 
several thousands of photons can be absorbed. For hot fullerenes, a broadening, as well as a shift of the vibrational resonance occurs depending on the temperature. In the experiment, such changes of the vibrational frequency can simply be compensated by the spectral width of the FELICE light beam [10]. The FEL intensity is set to a moderate value, just sufficient to ionize the molecules. Assuming that the fullerenes undergo thermionic emission, a given molecule must absorb typically 700 photons $(45 \mathrm{eV})$ in order to emit an electron within the observation time window in the $\mu$ s range [11]. Under these conditions, the probability of ionization at the beginning of the macropulse, where the internal energy of the molecule is low, is negligible. As a consequence, the uncertainty on the instant of ionization is smaller than the macropulse duration and estimated at $+/-1 \mu \mathrm{s}$. In Fig. 2(a), we show a typical electron momentum distribution measured at a FEL frequency of $525 \mathrm{~cm}^{-1}$. After angular integration, we obtain the electron kinetic energy spectrum. The temperature is extracted from a fitting procedure using Eq. (1). The spectrum obtained at $t_{m}=5 \mu$ s gives a temperature of $T=3000+/-200 \mathrm{~K}$. The time dependent temperatures obtained at FELICE are presented in Fig. 2(b). Importantly, they are in very good agreement with those obtained with UV-visible excitation also presented in the same figure. As described in Ref. [8] we have compared our measurements with a simulation of the time-dependent electron spectrum calculated within the Weisskopf framework, including thermionic emission, $\mathrm{C}_{2}$ emission, and radiative cooling channels, and assuming a broad internal energy distribution in accordance with the excitation process. Experimental temperatures are in very good agreement with this description (within a few percent). This has allowed us to determine that the emission rate is dominated by the dissociation rate [12]. In other words, the rate measured is essentially determined by the $\mathrm{C}_{2}$ loss rate approximated by $K(T)=K_{d}(T)=\omega_{d} \exp \left(-E_{d} / k_{B} T_{e}\right)$ where $E_{d}$ is the dissociation energy, $\omega_{d}$ the dissociation prefactor and the emission temperature $T_{e}$ is defined by $T_{e} \approx T_{\mu}(E-$ $\left.E_{d} / 2\right)$ (see Ref. [6]). We have determined $\left(E_{d}, \omega_{d}\right)=$ $\left(10.6 \mathrm{eV}, 10^{22} \mathrm{~Hz}\right)$ close to previous results [13]. This indicates that the UV-visible (ns) and far IR excitation lead to the same decay mechanisms and to the same temperature. This temperature is a property of the molecule and is independent of the excitation process itself.

In the following, we show that we can take advantage of the resonant character of the FELICE excitation to perform a mass selection of neutral species and therefore to study other fullerenes present in the molecular beam. This selectivity will further allow a precise characterization of the emission dynamics of the various fullerene species.

Time-of-flight spectra were recorded with a fullerene mixture while tuning the frequency of FELICE in the $600-850 \mathrm{~cm}^{-1}$ range. Drastic changes are observed in the ion mass distribution. In Fig. 3 the evolution of the ion
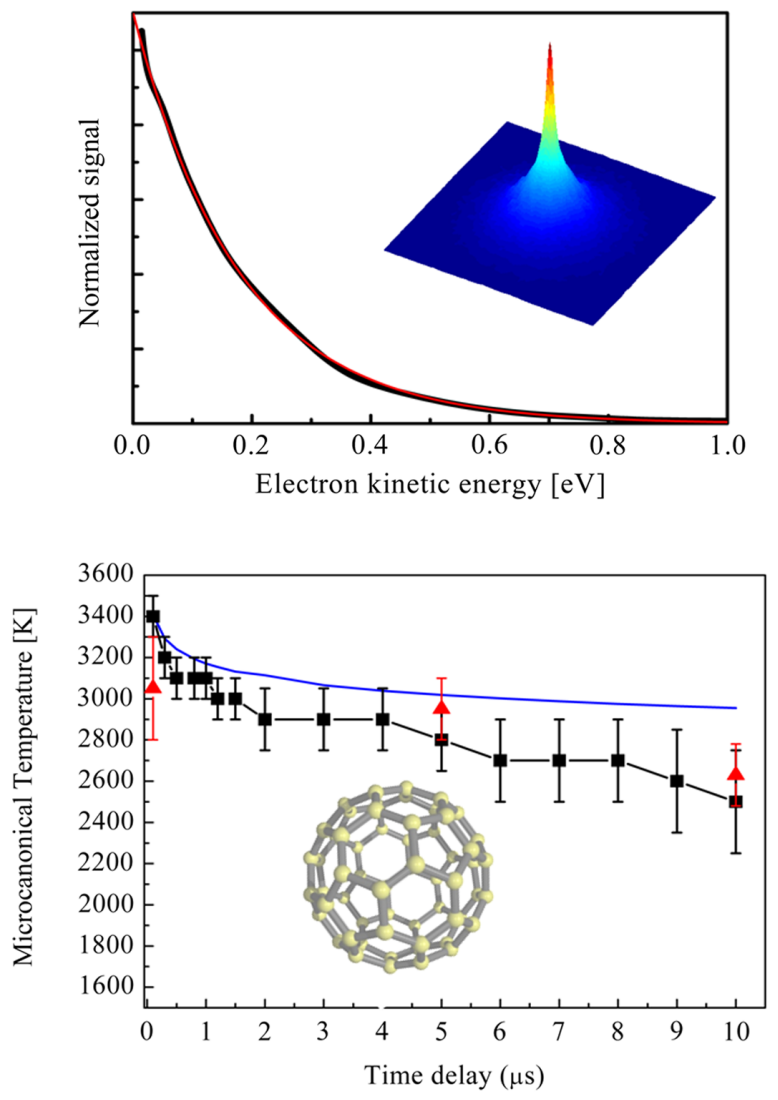

FIG. 2 (color online). Typical momentum electron image (inset) and corresponding kinetic energy spectrum measured with FELICE. (top, black line) A fitting procedure gives a direct measurement of the $\mathrm{C}_{60}$ microcanonical temperature at a welldefined time delay (bottom). Time evolution of the temperature of $\mathrm{C}_{60}$. Comparison between the results obtained at FELICE (red triangles) and measurements obtained with a $355 \mathrm{~nm}$ nanosecond laser (black squares Ref. [8]). The experimental data are well reproduced with Weisskopf's statistical theory of finite size systems including competing ionization and dissociation decay channels (blue line Ref. [8]).

signal intensity for several fullerene sizes $\mathrm{C}_{70}{ }^{+}, \mathrm{C}_{76}{ }^{+}, \mathrm{C}_{84}{ }^{+}$ is presented as a function of wavelength. $\mathrm{C}_{60}$ does not present any resonance in this spectral range [14], accordingly no signal is observed for $\mathrm{C}_{60}{ }^{+}$. The signal of individual species is strongly dependent on the wavelength, according to the vibrational mode excited by FELICE. As depicted in Fig. 3, this dependence can lead to a situation where only a single fullerene mass is ionized. For instance, at $680 \mathrm{~cm}^{-1}$ only $\mathrm{C}_{84}{ }^{+}$is produced. As a consequence, the mass selective ionization via a specific vibrational resonance allows us to study the decay of fullerenes of a specific mass even though all masses are present in the molecular beam. We have first selectively ionized the $\mathrm{C}_{70}$ fullerene using the specific resonance at $650 \mathrm{~cm}^{-1}$. Following the same procedure described above, we found the temperatures presented in Fig. 4(a). A comparison with the dissociation rate given in Ref. [11] is shown, using $E_{d}=9.7 \mathrm{eV}$ and $\omega_{d}=1.7 \times 10^{20} \mathrm{~Hz}$ which provides a very good 


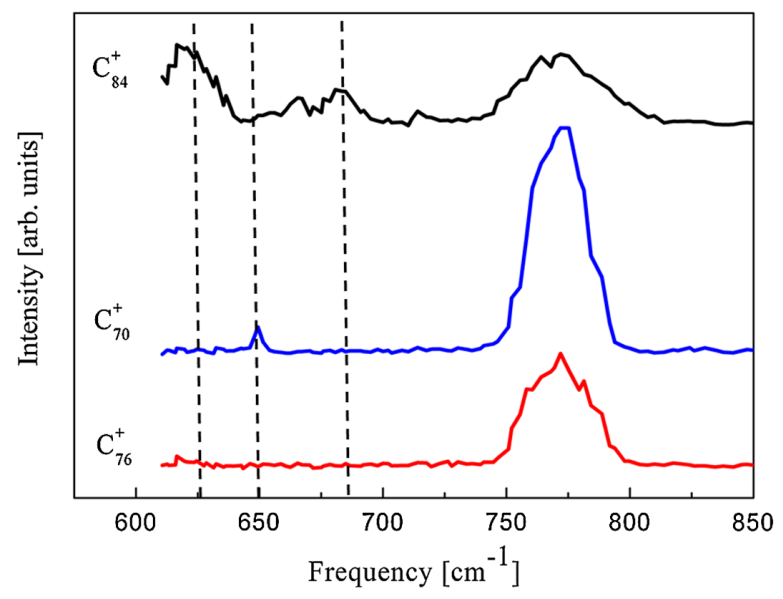

FIG. 3 (color online). Ion yield versus FEL wavelength for several ions produced from a fullerene mixture. The wavelength of the FEL is tuned continuously and excites resonances specific to a fullerene mass. IVR leads to a selective heating of the molecule that undergoes thermal electron ionization. Each vibrational resonance can be identified through the ion yield measurements. $\mathrm{C}_{84}{ }^{+}$(black curve), $\mathrm{C}_{70}{ }^{+}$(blue curve), $\mathrm{C}_{76}{ }^{+}$(red curve). The dashed lines indicate the frequencies used for the mass selection.

agreement with our experimental measurements. In the case of $\mathrm{C}_{60}$ and $\mathrm{C}_{70}$, both fullerenes could be produced as a pure molecular beam and the present results serve as a validation of the experimental protocol.

The case of $\mathrm{C}_{84}$ is more interesting from this point of view. As with $\mathrm{C}_{60}$ and $\mathrm{C}_{70}, \mathrm{C}_{84}$ is one of the magic fullerene sizes. Therefore, it has a stable cage structure and a high natural abundance. However, this system has been less studied than $\mathrm{C}_{60}$ and $\mathrm{C}_{70}$ and very little information is known on the $\mathrm{C}_{84}$ decay rates. Experiments performed by Gluch et al. [15] allowed extracting information on the $\mathrm{C}_{2}$ binding energy from measured kinetic energy release measurements, assuming a known value of the dissociative prefactor at $2 \times 10^{19} \mathrm{~Hz}$. They obtained $E_{d}=$ $8.8 \mathrm{eV}$ in agreement with tight binding calculations [16].

We have followed the exact same procedure described before in order to extract the time dependent temperature for $\mathrm{C}_{84}$. As shown in Fig. 4(b), we have chosen 2 vibrational resonances specific to $\mathrm{C}_{84}$ at 625 and $680 \mathrm{~cm}^{-1}$. On the same figure we have plotted the experimental results as well as the temperature calculated within the Weisskopf model including ionization and dissociation decay using parameters from Refs. [11,17]. The experimental temperatures appear independent of the chosen resonances within the error bars. Clearly the experimental temperatures are significantly lower than the theoretical prediction. In order to estimate the measured rate we set the binding energy to $8.8 \mathrm{eV}$. Using the Weisskopf calculation we extract from our measurements a prefactor of $2 \times 10^{22} \mathrm{~Hz}$. When $E_{d}$ is varied by $0.3 \mathrm{eV}, \omega_{d}$ varies by a factor of four. Let us notice that, the prefactor obtained for the $\mathrm{C}_{2}$ emission from $\mathrm{C}_{84}$ is

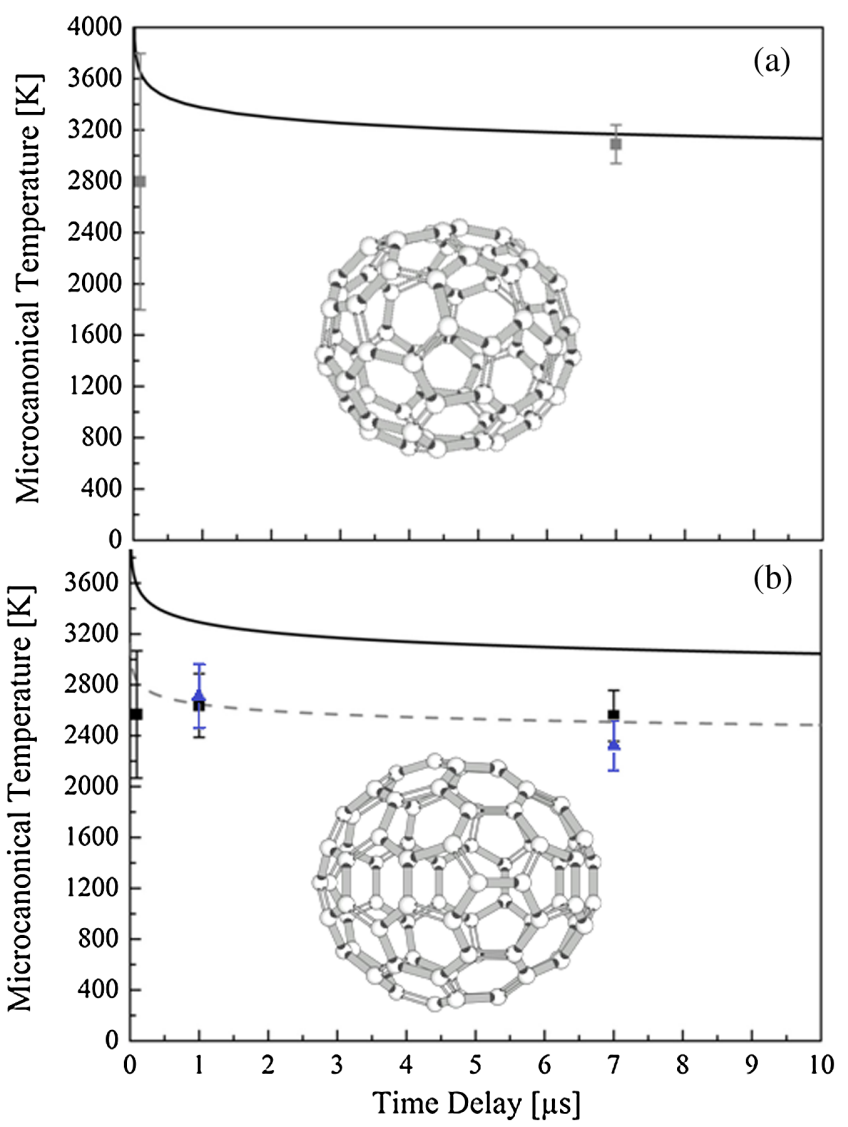

FIG. 4 (color online). (a) Time-dependent temperature of $\mathrm{C}_{70}$. The experimental results (gray square) are in very good agreement with the emission rate determined by $E_{d}=9.7 \mathrm{eV}, \omega_{d}=$ $1.7 \times 10^{20} \mathrm{~Hz}$ given in the literature (black curve). (b) Time dependent temperature of $\mathrm{C}_{84}$ measured at $625 \mathrm{~cm}^{-1}$ (blue triangle) and $680 \mathrm{~cm}^{-1}$ (black square) providing a direct measurement of the total rate. In the approximation where the rate is dominated by the dissociation and from the knowledge of the $\mathrm{C}_{2}$ binding energy $(8.8 \mathrm{eV})$ we have estimated the frequency prefactor to $2 \times 10^{22} \mathrm{~Hz}$ (dashed gray line), the black line indicates the rate from Ref. [11].

very close to the one obtained with our time-resolved imaging technique in the case of $\mathrm{C}_{60}$ (in that case the binding energy was equal to $10.6 \mathrm{eV}$ with $\omega_{d}=$ $1 \times 10^{22} \mathrm{~Hz}$ ). Let us emphasize that the theoretical prediction of the dissociation rate of a large polyatomic molecule is a very challenging task that requires a detailed description of the dissociation mechanisms; i.e., typical errors are of several orders of magnitude [18]. Our measurement provides a strong constraint to the estimation of the rates of the competitive channels and should help to improve theoretical descriptions.

As a conclusion, we have measured the time-dependent temperature of neutral fullerenes giving a direct insight into their decay mechanisms. We have validated our method using the known $\mathrm{C}_{60}, \mathrm{C}_{70}$ species and we have been able to obtain direct information on the dissociation of the $\mathrm{C}_{84}$ species that was less investigated in the literature. 
The present experiment demonstrates the possibility to study the stability and decay of individual neutral species that are otherwise difficult to obtain in a pure form, such as clusters that are only produced in a wide distribution of sizes. For instance, all systems that exhibit thermionic emission, such as the refractory metals niobium [19], tungsten, and tantalum [20] can be studied with this method. A necessary prerequisite is that information on the IR spectra is known, which can be obtained using various methods [21]. In the far-IR (or THz) domain it is possible to excite collective modes that are even more sensitive to very subtle changes in the geometry of the neutral systems. Therefore, it could also be possible to exploit this technique to study tautomers [22] or isomers. These possibilities will be investigated in a near future.

The authors would like to thank Dr. A. Fielicke for very fruitful discussion about the manuscript. We would like to acknowledge FP7 ELISA and ANR-10-BLAN-0428-01 for financial support. This work is part of the research program of the "Stichting voor Fundamenteel Onderzoek der Materie (FOM)", which is financially supported by the "Nederlandse organisatie voor Wetenschappelijk Onderzoek (NWO)". Support by MC-RTN "XTRA" (FP6-505138) is gratefully acknowledged.

*Corresponding author. franck.lepine@univ-lyon1.fr

[1] S. C. O'Brien, J. R. Heath, R. F. Curl, and R. E. Smalley, J. Chem. Phys. 88, 220 (1988).

[2] E. E. B. Campbell, K. Hansen, K. Hoffmann, G. Korn, M. Tchaplyguine, M. Wittmann, and I. V. Hertel, Phys. Rev. Lett. 84, 2128 (2000).

[3] K. Hansen, Philos. Mag. B 79, 1413 (1999).

[4] J. M. Bakker, V. J. F. Lapoutre, B. Redlich, J. Oomens, B. G. Sartakov, A. Fielicke, G. von Helden, G. Meijer, and A. F. G. van der Meer, J. Chem. Phys. 132, 074305 (2010).
[5] A. Eppink and D. H. Parker, Rev. Sci. Instrum. 68, 3477 (1997).

[6] F. Lépine and C. Bordas, Phys. Rev. A 69, 9 (2004).

[7] J. U. Andersen, E. Bonderup, and K. Hansen, J. Chem. Phys. 114, 6518 (2001).

[8] F. Lépine, B. Climen, M. A. Lebeault, and C. Bordas, Eur. Phys. J. D 55, 627 (2009).

[9] G. von Helden, I. Holleman, G. M. H. Knippels, A. F. G. van der Meer, and G. Meijer, Phys. Rev. Lett. 79, 5234 (1997).

[10] D. van Heijnsbergen, G. von Helden, B. Sartakov, and G. J. M. Meijer, Chem. Phys. Lett. 321, 508 (2000).

[11] G. von Helden, I. Holleman, A. J. A. van Roij, G. M. H. Knippels, A. F. G. van der Meer, and G. Meijer, Phys. Rev. Lett. 81, 1825 (1998).

[12] C. Lifshitz, Int. J. Mass Spectrom. 198, 1 (2000).

[13] B. Concina, S. Tomita, J. U. Andersen, and P. Hvelplund, Eur. Phys. J. D 34, 191 (2005).

[14] G. von Helden, I. Holleman, M. Putter, A. J. A. van Roij, and G. Meijer, Chem. Phys. Lett. 299, 171 (1999).

[15] K. Gluch, S. Matt-Leubner, O. Echt, B. Concina, P. Scheier, and T. D. Märk, J. Chem. Phys. 121, 2137 (2004).

[16] B. L. Zhang, C. H. Xu, C. Z. Wang, C. T. Chan, and K. M. Ho, Phys. Rev. B 46, 7333 (1992).

[17] H. Steger, J. Holzapfel, A. Hielscher, W. Kamke, and I. V. Hertel, Chem. Phys. Lett. 234, 455 (1995).

[18] K. Hansen, E. E. B. Campbell, and O. Echt, Int. J. Mass Spectrom. 252, 79 (2006).

[19] V. J. F. Lapoutre, B. Redlich, J. Oomens, A. F. G. van der Meer, M. Haertelt, A. Fielicke, G. Meijer, and J.M. Bakker (to be published).

[20] A. Amrein, R. Simpson, and P. Hackett, J. Chem. Phys. 94, 4663 (1991).

[21] A. Fielicke, A. Kirilyuk, C. Ratsch, J. Behler, M. Scheffler, G. von Helden, and G. Meijer, Phys. Rev. Lett. 93, 023401 (2004).

[22] A. Fielicke, C. Ratsch, G. von Helden, and G. Meijer, J. Chem. Phys. 122, 091105 (2005). 